\section{A school-based oral health intervention in East London: the Happy Teeth fluoride varnish programme}

\author{
P. Evans, ${ }_{1}^{1}$ N. Pearson ${ }^{2}$ and D. Simons ${ }^{* 3}$
}

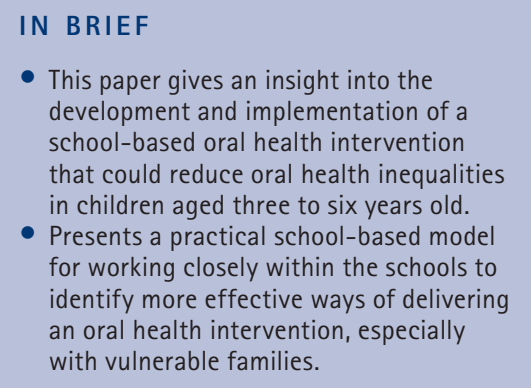

\begin{abstract}
Background The Community Dental Services of Barts Health NHS Trust in City and Hackney and Tower Hamlets PCTs in East London have provided a school-based oral health intervention since 2009. Objective The aim of this paper is to present the programme development, outcomes and evaluation. Subjects and methods The programme consists of fluoride varnish applications linked to school dental screenings for three to six-year-olds, combined with oral health promotion for parents/carers. An outreach linkworker works closely with schools to help identify and support vulnerable families into the programme. Results In the first year of the programme 160 of the target children (42\%) had one FV application and 81 children (21\%) had two applications. In the second year 149 children (39\%) had one FV application, and $113(29 \%)$ had two applications. Amendments to the protocol increased programme participation in the third year, with 1,822 of the target children (61\%) having one FV application and 1,586 (53\%) having two applications. Conclusions The programme proved acceptable to the school staff, participating parents/carers and children. The Happy Teeth programme is proposed as a model for school-based fluoride varnish programmes.
\end{abstract}

\section{INTRODUCTION}

The Happy Teeth fluoride varnish programme consists of fluoride varnish (FV) applications linked to school screenings, together with oral health promotion sessions for their parents/ carers. This paper reports on the development of the programme protocol along with uptake of dental screening, fluoride varnish application and qualitative process evaluation conducted with school staff and parents/carers of participating children.

Dental caries is a preventable but common occurrence in children. Childhood caries can affect quality of life and growth through discomfort, pain, difficulty in eating, sleeplessness, loss of confidence, poor concentration at school, damage to underlying permanent teeth and may expose children to the risks associated with

Senior Dental Officer, ${ }^{2}$ Clinical Director, ${ }^{3}$ Assistant Clinical Director, Barts Health NHS Trust, Community Dental Services, 3rd floor Block B, St Leonard's Primary Care Centre, Nuttall Street, London, N1 5LZ

${ }^{*}$ Correspondence to: Debra Simons

Email: Debra.Simons@bartshealth.nhs.uk

\section{Online article number E14}

Refereed Paper - accepted 10 July 2013

DOI: $10.1038 /$ sj.bdj.2013.997

${ }^{\circ}$ British Dental Journal 2013; 215: E14 general anaesthesia (GA). ${ }^{1,2}$ Caries is closely associated with social deprivation resulting in large geographical and socioeconomic inequalities across the UK. ${ }^{3}$ Caries in five-year-old children is considered an important outcome indicator for oral health by the Department of Health (DH) and is included in the Public Health Outcomes Framework. ${ }^{4}$ The East London boroughs of City and Hackney $(\mathrm{CH})$ and Tower Hamlets (TH) are two of the most deprived boroughs in England. ${ }^{5}$ Data from national surveys demonstrate high levels of dental decay in the school age population of $\mathrm{CH}$ and $\mathrm{TH}$. In a sample of five-year-old children, the average number of treated and untreated teeth in those children who have experience of dental decay was 4.26 in $\mathrm{CH}$ and 4.48 in $\mathrm{TH}$. This compares unfavourably with the average for London (3.88) and England as a whole (3.45). ${ }^{6}$ Uptake of dental services by children and young adults is $46 \%$ in $\mathrm{CH}$ and 55\% in $\mathrm{TH}$ - which is lower than the London average of $67 \%$ and England average of 70\%., ${ }^{7,8}$ Therefore any preventive strategy aimed at families attending dental practices will miss many young children.

Choosing better oral health ${ }^{9}$ set out the evidence base for effective prevention of dental caries and other dental diseases, highlighting the need for multidimensional programmes and avoiding traditional and outdated health education approaches. Delivering better oral health: an evidence-based toolkit for prevention ${ }^{10}$ gives guidance to dental teams on how to prevent oral disease. Twice yearly applications of FV in children have been shown to reduce dental decay in primary teeth by $33 \%$ and $46 \%$ in permanent teeth. ${ }^{11}$ The toolkit recommends that all children aged three years through to adulthood have FV applied professionally twice yearly, and three to four times a year for those children who are considered more vulnerable to caries development. ${ }^{10}$

Since 1918 the Salaried Dental Services has carried out school dental screenings, ${ }^{12}$ however, screening on its own has not been an effective intervention to promote dental attendance for those children who require treatment or reduce levels of active caries. ${ }^{13}$ The DH has recommended that individual Primary Care Trusts (PCTs) should decide whether or not to continue school dental screening in their area. ${ }^{14}$ Since 2006, in England, Wales and Northern Ireland, positive consent has been required for dental screenings and surveys, which resulted in a decrease in participation, and 
Monaghan et al. ${ }^{15}$ suggest that parents of children with caries are less likely to participate. Schools are recognised as an ideal setting ${ }^{16,17}$ and 'potential entry point' ${ }^{18}$ for a health promotion intervention. A school-based programme may help to reduce health inequalities by addressing barriers to accessing preventive dental care. ${ }^{19}$ School programmes do not need to rely on parental attendance once consent has been obtained for their child to participate. Additionally, schools can help support vulnerable children - for example those with special needs - into programmes through their knowledge of individual family social background, language and culture. ${ }^{20}$ The UK government endorses the role of the school in supporting the health and well-being of children. The new Healthy Schools toolkit recommends evidence-based health interventions, such as FV application, to ensure schools put in place the most appropriate services to meet the needs of pupils. ${ }^{17}$ The Scottish dental preventive programme Childsmile has successfully incorporated a schoolbased FV application element. ${ }^{21}$ Utilising extended duty dental nurses (EDDNs) has been shown to maximise the cost effectiveness of community-based FV programmes. ${ }^{22}$

Watt et $a l .{ }^{23}$ highlighted the need to evaluate health promotion interventions for impact and value, specifically for: developing good practice, making best use of limited resources, providing feedback to staff and participants, and informing policy development. ${ }^{24}$ They suggest quantitative and qualitative evaluation, which 'needs to be appropriate to the intervention and timescales involved in the programme.. ${ }^{23}$

$\mathrm{CH}$ and TH PCTs commissioned the Community Dental Services (CDS) to provide a screening FV programme for children aged three to six years in a school setting. The aim of this paper is to present the programme's development, outcomes and evaluation.

\section{METHODS}

At the beginning of 2009 (Year 1), one school in $\mathrm{CH}$ and two in $\mathrm{TH}$ with the highest caries rates in five-yearold children were identified by the PCT for participation in the programme. ${ }^{6}$ Local GDPs were kept informed of the programme by letter and PCT newsletter.
The programme aimed to work closely with the head teachers of local schools to secure the school's participation. A key school contact was identified by the head teacher to support the dental team and help maximise uptake of the programme, for example a home-school liaison worker or a member of the teaching staff. Flexibility and understanding of the competing priorities and pressures in schools was necessary when timetabling the different parts of the programme. School class lists ensured that target numbers for each class were known. Information and consent forms were distributed to schools to send home with every target child. CDS outreach linkworkers arranged and advertised parent information sessions at the schools before the clinical element of the programme began. These sessions with parents were planned to explain the project and answer any questions, as well as to provide important information about how to prevent oral disease and access care. Vulnerable and hard to reach families, identified by the school as needing extra support to enter the programme, were assisted in the process by a home school liaison worker. All queries from parents were responded to by the linkworker either face-to-face or on the telephone.

Advice was sought from the $\mathrm{DH}$, the Medical Protection Society and the PCT in developing the informed consent process and the relevant medical history questions. Separate consent forms were used for the screening and for the FV application. Parents were advised to complete both consent forms if they wished their child to have the FV application, as their child required a screening before the dentist wrote the fluoride prescription. Parents were advised in the FV information sheet of the presence of alcohol in the varnish used (Duraphat ${ }^{\circledR}$ ), and a statement of its acceptability was included from the Islamic Sharia Council and a prominent local Orthodox Jewish rabbi. All parents of children in nursery, reception and year one (age three to six years old) in the selected schools were offered the opportunity to participate in the programme. Children were excluded from the FV application if they suffered from asthma or any other allergy; received any fluoride supplements other than toothpaste; or received FV from their own dentist. Children were also excluded on the day of the application if they were systemically unwell, uncooperative, had a sore mouth, dental abscess or sinus. Parents were offered the opportunity to attend their child's appointment. Medical histories were updated before the second varnish application.

Before a clinical session, the school premises were risk assessed using a pro forma developed for the purpose. The screening and FV application sessions were conducted following a strict protocol to ensure the smooth and safe running of the programme, and that only correctly identified children participated. The clinician was responsible for collecting the children from their classrooms. Care was taken to approach the most appropriately placed school staff to identify individual children correctly. A dentist undertook the dental screening session, following which a prescription for two FV applications was written in the child's record card. Screening outcomes were documented. Parents were informed in writing if their child had a dental treatment need identified or further examination or advice was indicated. A list of local NHS dental practices was included in the letter, plus contact details for the CDS if advice or information was required. A hygienist or EDDN undertook the fluoride application sessions within two weeks of the screening session in a mobile dental unit parked in the school playground. PCT infection control guidelines were followed. Following a clear extra- and intraoral assessment $0.25 \mathrm{ml}$ FV was applied to all surfaces of posterior molars, canines and upper incisors. The child then received a free toothbrush and toothpaste. Parents were informed in writing that their child had received a FV application and that they should avoid soft foods and were not to brush their child's teeth that day. They were also advised to inform their child's regular dentist of the application at school. Parents were informed in writing if, and why, their child was excluded from the FV application. School staff were advised not to allow children to eat or drink for half an hour following the application, and care was taken to timetable application sessions to allow for this.

The dental team returned to each school six months later for the second FV application. Updated medical history letters were followed up with a telephone call to complete verbally if necessary. Hygienists and EDDNs applied the FV. Children were 
identified for follow-up who still had a dental treatment need as diagnosed at the initial screening, and this part of the programme is reported elsewhere. ${ }^{25}$ The programme was repeated for the target age group (age three to six years) in the same schools in 2010/11 (Year 2), and in 2011/12 (Year 3). Additionally, in Year 3 a further 17 schools were included in the programme - 4 in $\mathrm{CH}$ and 13 in $\mathrm{TH}$ - for the same target age group. The protocol was amended in Year 3 to improve programme uptake - these amendments are detailed in the discussion.

\section{DATA COLLECTION AND EVALUATION}

Anonymised quantitative data was collected: target number of children, number screened, number consenting to FV, number excluded, and number receiving FV. Qualitative process evaluation was conducted by the dental linkworker to assess programme acceptability. This was conducted by telephone or face-to-face semi-structured interviews with a convenience sample of parents/carers of children who had participated. The parent/carer evaluation questions, which were not validated, were developed around the following themes: adequacy of parent information before the FV application, adequacy of information after the FV application, the parent's perception of their child's experience of the FV application, and likelihood of consent for the following year's programme. Written semi-structured feedback was collected at the end of Year 3 from the head teachers at the school around the following themes: what worked well in the programme, how the programme could be improved, satisfaction with programme organisation and correspondence; and satisfaction with the dental team at the school.

\section{RESULTS}

All selected schools agreed to participate in the programme. For Year 1 (2009/10; three schools), from a target number of 385 children, 274 (71\%) children were screened and 257 (67\%) consented to the FV application. However, 97 children, (38\% of those who consented), were then excluded: 67 for medical reasons, 3 excluded on the day (due to a dental abscess or sore mouth) and 27 were absent or refused the application. One hundred and sixty of

Table 1 Parent responses to evaluation questions at end of Year $2(2010 / 11) ;(n=56)$

\begin{tabular}{|l|l|l|l|}
\hline & \multicolumn{2}{|l}{ Frequency of Response } \\
\hline & $\begin{array}{l}\text { Positive } \\
\text { response }\end{array}$ & $\begin{array}{l}\text { Neutral } \\
\text { response }\end{array}$ & $\begin{array}{l}\text { Negative } \\
\text { response }\end{array}$ \\
\hline Parent information before application & $54(96 \%)$ & $0(0 \%)$ & $2(4 \%)$ \\
\hline Parent information after application & $56(100 \%)$ & $0(0 \%)$ & $0(0 \%)$ \\
\hline Child experience of varnish application & $46(82 \%)$ & $7(13 \%)$ & $3(5 \%)$ \\
\hline Parent consent to application the following year & $52(93 \%)$ & $4(7 \%)$ & $0(0 \%)$ \\
\hline
\end{tabular}

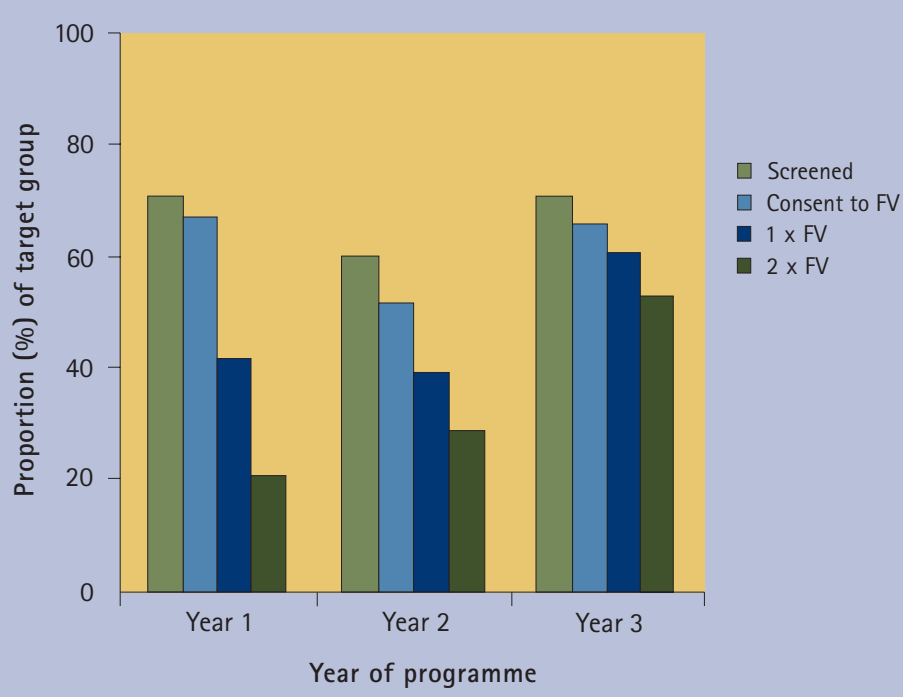

Fig 1 Proportion of target group that was screened, consented to fluoride application (FV) had at least one fluoride application [1 $\times \mathrm{FV}]$, and had two fluoride applications $[2 \times \mathrm{FV}]$ over all three years

the target children (42\%) had one FV application and 81 children (21\%) had two applications (Fig. 1).

For Year 2 (2010/11; three schools), from a target number of 385 children, $231(60 \%)$ were screened and 52\% ( $\mathrm{n}=202$ ) consented for the FV application. However, 53 children, (26\% of those who consented), were then excluded: 40 for medical reasons, 3 on the day (due to a dental abscess or sore mouth) and 10 were absent or refused the application. One hundred and forty-nine children (39\%) had one FV application, and 113 (29\%) had two applications (Fig. 1).

For Year 3 (2011/12; 20 schools), from a target number of 2,990 children, 2,114 (71\%) had a dental screening and $1,984(66 \%)$ consented for the FV application. However, 162 children, (8\% of those who consented), were excluded: 68 for medical reasons, 33 excluded on the day (due to a dental abscess or sore mouth) and 61 refused the FV application. Sixty-one percent $(n=1,822)$ of the target children had one FV application and 53\% $(\mathrm{n}=1,586)$ had two applications (Fig. 1).
Parental responses to the semi-structured interview at the end of Year $2(2010 / 11)$ are summarised in Table 1. A convenience sample of 56 parents was interviewed. Fifty-four parents (96\%) were happy about the amount of information provided before the FV application, but two parents (4\%) wanted more information. All parents were happy with the post-operative information provided after their child had received the varnish application. Most parents, $82 \%(n=46)$, reported that their child's experience of the application was positive. Seven (13\%) of parents were neutral about their child's experience and three (5\%) of parents reported that their child had had a negative experience. Most parents, 93\% ( $n=52$ ), wanted their child to participate in the programme in the following year, while the remaining $2 \%(n=4)$ of parents were unsure.

Evaluation forms from $4(20 \%)$ of the 20 schools were returned at the end of Year 3. Two schools commended use of the mobile dental unit and two evaluators commended the parent information sessions. One evaluator praised the staff 
and one appreciated the lack of need for school involvement. Suggestions for improvement included: letting teachers distribute toothbrush packs at the end of the day to avoid classroom chaos, simplifying consent forms, more parent workshops, and ensuring school staff were allocated to help teams. Evaluators scored 'good', 'very good' or 'excellent' for satisfaction with organisation and correspondence, and 'very good' or 'excellent' for satisfaction with clinical staff.

\section{DISCUSSION}

Although school dental screening has been discredited as a method of reducing active caries levels or increasing dental attendance, ${ }^{13}$ when combined with other evidence-based interventions it may be effective in reducing dental health inequalities in school children. ${ }^{26}$ School FV programmes are more effective in highrisk populations, ${ }^{27}$ such as East London, and this programme additionally targeted children identified with caries for a further intervention, which is reported elsewhere. ${ }^{25}$ Using dentists to carry out a screening before prescribing FV ensured that dental caries was diagnosed and recorded as early as possible. Children with an urgent dental treatment need were followed up at the second varnish application by EDDNs and hygienists, who were given appropriate training to recognise and highlight to parents/carers a continuing treatment need.

The Health Development Agency has emphasised the importance of tailoring and targeting public health interventions so that not only the most socially disadvantaged groups benefit, but also those in and below the middle spectrum of the health and social gradient to avoid increasing and elongating this gradient. ${ }^{28}$ Although $\mathrm{CH}$ and $\mathrm{TH}$ are two of the most deprived boroughs in England, not all families are socioeconomically disadvantaged. No attempt was made in this programme to explore the demographic profile of the children who participated and those who did not, and this could be explored in a further study using, for example, free school meal eligibility as a deprivation indicator, or home postcodes to identify deprivation indices. Hardman et al. ${ }^{29}$ have suggested that less deprived children may be more likely to participate in FV programmes, thereby reducing their impact on dental health inequalities.

As this programme is school-based, it ensures that children who do not routinely access dental care have the opportunity to participate in a dental preventive intervention. Using the school's knowledge of an individual child's family and social background, as well as the school's position and authority to support targeted parents, this programme could access information and resources normally unavailable to dental services. This close partnership with schools may be a strategy to remove barriers to accessing dental care for vulnerable children. ${ }^{30}$ Use of outreach linkworkers who forged strong links with the schools ensured that the programme remained prominent and schools had up-to-date knowledge of dental care pathways. The use of hygienists and EDDNs to apply FV in a community setting such as a school offers an opportunity for skill mix in dentistry ${ }^{31}$ and maximises cost effectiveness. $^{22}$

Unlike other studies of school-based FV programmes for primary school aged children, baseline and incremental caries data were not collected. ${ }^{27,29,32-35}$ This was because the programme was established to develop an acceptable, effective and sustainable process to deliver an evidencebased intervention. ${ }^{36}$ The programme was dynamic, and amendments to the original protocol following the dental team's experiences and feedback from schools and parents improved the uptake of FV and cost effectiveness in Year 3. A combined single consent form was developed for both screening and FV application, and parents were routinely telephoned in order to complete partially filled-in forms. This meant that fewer consenting children were excluded due to missing information. Children were only excluded on medical grounds if they were at risk of an allergic reaction to colophony (a constituent of Duraphat ${ }^{\circledR}$ ), and this was ascertained by a history of hospitalisation due to severe asthma or allergy, or allergy to sticking plaster. ${ }^{37}$ To reduce the dropout rate before the second application, parents were advised only to contact the dental team if their child's medical history had changed rather than having to return the repeat medical questionnaire to school. The uptake of consents for the FV in Year 3, 66\% of the target children, was similar to that of Year 1 (67\%), however, the proportion of those children who went on to receive at least one FV application was increased from 42\% (Year 1) and 39\% (Year 2) to 61\% in Year 3. Similarly, the proportion of children who received two applications of FV was increased from $21 \%$ in Year 1, to 29\% in Year 2, to 53\% in Year 3. Although participation in the programme increased over the three years, $39 \%$ of target children did not receive any fluoride varnish in the final year. Nursery and reception children from Year 1 had an opportunity to also participate in the subsequent year(s), but no attempt was made to follow individual children's participation through the programme year on year, which is a limitation of the evaluation.

Screening and varnish application sessions were adapted to run more effectively over the three years. If the school could facilitate, applications were undertaken in a suitable room rather than in a mobile dental clinic to reduce time and the cost of programme provision. Children lay on a low table covered by a mat; an angle poise lamp provided illumination. The clinician sat behind the child on a low chair. A dentist applied the first FV application at the time of the screening. Parents were not invited to attend the screening or FV application of their child as this proved very time consuming for the clinician, however, they were permitted to attend if they requested to do so. All queries from parents continued to be responded to by the linkworker.

Programme information and consent forms were not translated into other languages, and both parental language and literacy barriers may have had an impact on uptake in schools serving the culturally diverse community of East London. Although use was made of school translation services, this was not always available. A Bengali-speaking programme administrator was employed in Year 3 and this helped improve uptake in schools with a high Bengali-speaking population, although this was not fully evaluated. Furthermore, the alcohol content of the FV product used in the programme may have influenced parental decision to consent, particularly for Muslim families. This may explain why each year a group of parents only consented to the screening element of 
the programme, which could be explored in future evaluation.

Parents/carers of participating children were happy with programme information, and were willing to consent for the following year's programme.

These parents perceived that their children found the FV application experience acceptable. However, sampling bias may have affected the results: this was a convenience sample and interviewers only sampled consenting parents whose children had received the varnish application, ignoring the views of parents who had not consented to their child participating in the programme. To ensure that barriers to programme participation are addressed, this could be explored in further research. Future programme process evaluation could also include feedback from dental teams and directly from participating children. Uptake and evaluation of parent sessions would also be valuable in assessing their impact on engagement with the programme and other dental services. The feedback response rate from the schools was low and in future research verbal feedback may be more appropriate.

\section{CONCLUSION AND RECOMMENDATIONS}

This report has demonstrated that a school-based FV application programme is feasible and acceptable to schools, parents and children in this community. Uptake of the intervention can be improved by working closely with the schools to identify more practical and effective ways of delivering the programme. Dental outreach linkworkers can use school support systems to enable vulnerable families to access the programme. The Happy Teeth programme could be used as a model for other school-based FV programmes, and this can be tested in different communities.

The authors would like to acknowledge Dr Ursula Bennett and Tricia Wallace for their vision and commitment in establishing the Happy Teeth programme. They would also like to thank $\mathrm{Dr}$ Grammati Sarri for her comments on earlier versions of this paper.

1. Nuttall N, Harker R. Impact of oral health: children's dental health in the United Kingdom. London: Office of National Statistics, 2004. Online article available at http://www.ons.gov.uk/ons/guide-method/ method-quality/specific/health-methodology/ dental-health/dental-health-of-children/index.html (accessed July 2013)
2. Sheiham A. Dental caries affects body weight, growth and quality of life in pre-school children. Br Dent J 2006; 201: 625-626.

3. British Dental Association. Oral health inequalities policy. London: BDA, 2009. Online policy available at http://www.bda.org/dentists/policy-campaigns/ public-health-science/public-health/oral-healthinequalities.aspx (accessed July 2013).

4. Department of Health. Public health outcomes framework 2013 to 2016. London: DH, 2012. Online article available at https://www.gov.uk/government/ publications/healthy-lives-healthy-peopleimproving-outcomes-and-supporting-transparency (accessed July 2013)

5. Department for Communities and Local Government English indices of deprivation 2010. London: Crown Copyright, 2011. Online article available at https:// www.gov.uk/government/publications/englishindices-of-deprivation-2010 (accessed July 2013).

6. Public Health England. Results of five year old children survey, 2007/08. Public Health England, 2009. Online article available at http://www. nwph.net/dentalhealth/survey-results.aspx?id=1 (accessed July 2013)

7. City and Hackney Primary Care Trust. City and Hackney health and wellbeing profile, 2011/12: our joint strategic needs assessment. London: City and Hackney Primary Care Trust, 2012. Online article available at http://www.hackney.gov.uk/ Assets/Documents/City-and-Hackney-Health-andWellbeing-Profile-2011-12.pdf (accessed July 2013).

8. Tower Hamlets Primary Care Trust. Joint strategic needs assessment: an overview of health and wellbeing in Tower Hamlets, 2010-2011. London: Tower Hamlets Primary Care Trust, 2011. Online article available at http://www.towerhamlets.gov. uk/lgsl/701-750/732_jsna.aspx (accessed July 2013).

9. Department of Health. Choosing better oral health: an oral health plan for England. London: $\mathrm{DH}$, 2005. Online article available at http://webarchive. nationalarchives.gov.uk/20130107105354/http:// www.dh.gov.uk/en/Publicationsandstatistics/ Publications/PublicationsPolicyAndGuidance/ DH_4123251 (accessed July 2013).

10. Department of Health. Delivering better oral health: an evidence-based toolkit for prevention. 2nd ed. London: DH 2009. Online article available at http://webarchive.nationalarchives. gov.uk/20130107105354/http://www.dh.gov. uk/en/Publicationsandstatistics/Publications/ PublicationsPolicyAndGuidance/DH_102331 (accessed July 2013).

11. Marinho V C, Higgins J P, Logan S, Sheiham A. Fluoride varnishes for preventing dental caries in children and adolescents. Cochrane Database Syst Rev 2002; 3: CD002279.

12. Department of Education. The education act London: HMSO, 1918

13. Milsom K M, Blinkhorn A S, Worthington $\mathrm{H}$ et al. The effectiveness of school dental screening: a cluster-randomized control trial. J Dent Res 2006: 85: 924-928.

14. Department of Health. Dental screening (Inspection) in schools and consent for undertaking screening and epidemiological surveys. London: DH, 2007. Online article available at http://webarchive. nationalarchives.gov.uk/20130107105354/http:// www.dh.gov.uk/en/Publicationsandstatistics/ Publications/PublicationsPolicyAndGuidance/ DH_064173 (accessed July 2013).

15. Monaghan N P, Jones S J, Morgan M Z. Do parents of children with caries choose to opt out of positive consent dental surveys in Wales? Br Dent J 2011; 210: E1.

16. World Health Organization. Promoting health through schools: the WHO global school initiative. Geneva: WHO, 1986.

17. Department for Education. Healthy schools. Online information available at http://www.education.gov. uk/schools/pupilsupport/pastoralcare/a0075278/ healthy-schools. (accessed July 2012).

18. Blas E, Kurup A S. Equity, social determinants and public health programmes. Geneva: WHO, 2010. Online article available at http://whqlibdoc.who. int/publications/2010/9789241563970_eng.pdf (accessed July 2012).

19. Finch H, Keegan J, Ward K, Sanyal Sen B. Barriers to the receipt of dental care: a qualitative study. London: Social and Community Planning Research, 1988.

20. Macpherson L M, Ball G E, Brewster L et al. Childsmile: the national child oral health improvement programme in Scotland. Part 1: establishment and development. Br Dent J 2010; 209: 73-78.

21. Turner S, Brewster L, Kidd J et al. Childsmile: the national child oral health improvement programme in Scotland. Part 2: monitoring and delivery. Br Dent J 2010; 209: 79-83.

22. Jones $C M$, Walker $A$. The role of extended duties dental nurses in applying fluoride varnish for the prevention and control of dental caries. Dent Update 2010; 37: 549-554.

23. Watt $R$, Fuller, $S$, Harnett, $R$, Treasure E, StillmanLowe C. Oral health promotion evaluation - time for development. Community Dent Oral Epidemiol 2001; 29: 161-166.

24. Blinkhorn A. Evaluation and planning of oral health promotion programmes. In Schou L, Blinkhorn A (eds) Oral health promotion. pp 249-270. Oxford: Oxford University Press, 1993.

25. Simons D, Pearson N, Evans P. A pilot of a school-based dental treatment programme for vulnerable children with possible dental neglect: the Back2School programme. Br Dent J 2013; 215: E15

26. Davies G, Bridgman C. Improving oral health among schoolchildren - which approach is best? Br Dent J 2011; 201: 59-61.

27. Arruda A O, Kannan R S, Inglehart M R, Rezende C T, Sohn W. Effect of 5\% fluoride varnish application on caries among school children in rural Brazil: a randomized controlled trial. Community Dent Oral Epidemiol 2012; 40: 267-276.

28. Health Development Agency. The evidence of effectiveness of public health interventions - and the implications. Health Development Agency, 2004. Online article available at http://www.nice.org.uk/ nicemedia/documents/CHB1-public-health.pdf (accessed July 2013)

29. Hardman M C, Davies G, Duxbury J T, Davies R M. A cluster randomised controlled trial to evaluate the effectiveness of fluoride varnish as a public health measure to reduce caries in children. Caries Res 2007; 41: 371-376.

30. Sarri G, Marcenes W. Child dental neglect: is it a neglected area in the UK? Br Dent J 2012; 213: 103-104.

31. Milsom K M, Tickle M. Preventing decay in children: dare we risk the 'risk assessment' model in practice? Br Dent J 2010; 209: 159-160.

32. Zimmer S, Robke F J, Roulet J F. Caries prevention with fluoride varnish in a socially deprived community. Community Dent Oral Epidemiol 1999; 27: 103-108.

33. Autio-Gold J T, Courts F. Assessing the effect of fluoride varnish on early enamel carious lesions in the primary dentition. J Am Dent Assoc 2001; 132: 1247-1253.

34. Ersin N K, Eden E, Eronet N, Totu FI, Ates M. Effectiveness of 2-year application of schoolbased chlorhexidine varnish, sodium fluoride gel, and dental health education programmes in high-risk adolescents. Quintessence Int 2008; 39: e45-51.

35. Milsom K M, Blinkhorn A S, Walsh A clusterrandomized controlled trial: fluoride varnish in school children. J Dent Res 2011; 90: 1306-1311.

36. Weightman L, Ellis S, Cullum A, Sander L, Turley R. Grading evidence and recommendations for public health interventions: developing and piloting a framework. Health Development Agency, 2005.

37. Scottish Dental Clinical Effectiveness Programme. Prevention and management of dental caries in children: dental clinical guidelines. Edinburgh: SDCEP, 2010. Online guidelines available at http:// www.sdcep.org.uk/?0=2858 (accessed July 2013). 